\title{
Interactions between spherical nanoparticles optically trapped in Laguerre-Gaussian modes
}

\author{
David S. Bradshaw and David L. Andrews \\ Nanostructures and Photomolecular Systems, School of Chemical Sciences, University of East Anglia,
} Norwich NR4 7TJ, UK

\begin{abstract}
Received April 28, 2005; revised manuscript received June 21, 2005; accepted June 25, 2005
When a Laguerre-Gaussian (LG) laser mode is used to trap nanoparticles, the spatial disposition of the particles about the beam axis is determined by a secondary mechanism that engages the input radiation with the interparticle potential. This analysis, based on the identification of a range-dependent laser-induced energy shift, elicits and details features that arise for spherical nanoparticles irradiated by a LG mode. Calculations of the absolute minima are performed for LG beams of variable topological charge, and the results are displayed graphically. It is shown that more complex ordered structures emerge on extension to threeand four-particle systems and that similar principles will apply to other kinds of radially structured optical mode. (C) 2005 Optical Society of America
\end{abstract}

OCIS codes: $020.5580,140.7010,220.4880,260.2110,270.5580,290.5890$.

Recently, there has been an increasing focus on the implementation of optical tweezer instrumentation with Laguerre-Gaussian (LG) modes, producing effects that have become known as optical spanners. ${ }^{1}$ As well as a rotational influence, such beams have transverse structures that offer novel possibilities for particle trapping. While there is a considerable body of work on the forms of optomechanical coupling involved in optical tweezers and spanners, in which incoming laser light exerts forces on individual particles, it is less well known that there exist entirely distinct optically induced interparticle interactions. ${ }^{2,3}$ In detail, the mechanism involves the annihilation of a real laser input photon at one particle and the stimulated emission of an equivalent real photon at the other, with a virtual photon acting as mediator between the two particles. Two representative time-ordered diagrams are given in Fig. 1. The aim of this Letter is to identify distinctive features that arise for spherical nanoparticles irradiated by a LG beam, especially those that differ from the effects of conventional Gaussian light (the latter being the subject of other recent work $\left.^{4}\right)$. This purpose is achieved by determining the laser-induced interparticle energy shift for incoming LG modes-modes characterized by two integers, $l$ and $p$, the former being the topological charge and $(p+1)$ denoting the number of radial nodes-using a recently completed QED formulation of this type of photon-matter interaction. ${ }^{5}$ Although LG modes have recently attracted considerable attention with regard to their orbital angular momentum, ${ }^{6,7}$ an attribute relating to a helically twisted wavefront structure, this is not a feature that plays a part in the underlining phenomena discussed below, for reasons that will emerge.

Two-particle system. Consider a pair of identical spherical nanoparticles (A and B) optically trapped in an annular intensity region of a LG beam with $p=0$, i.e., an optical vortex with one radial node at the beam center (Fig. 2). Both particles are assumed to have the same displacement $\zeta$ along the beam propagation direction, i.e., they are contained within a cross section of the beam, and they are situated at the same distance from the beam center, $r$, while their azimuthal relative displacement angle, $\Delta \psi=\psi_{B}$ $-\psi_{A}$, is variable. The laser-induced energy shift, $\Delta E_{\mathrm{AB}}$, for this configuration has been determined in earlier work ${ }^{8,9}$ and is given by

$$
\begin{aligned}
\Delta E_{\mathrm{AB}}= & \left(\frac{I f_{l p}^{2} \alpha^{2}}{4 \pi \epsilon_{0}^{2} c A_{l p}}\right)\left[( 1 - 3 \operatorname { s i n } ^ { 2 } \phi ) \left(\frac{\cos k R}{R^{3}}\right.\right. \\
& \left.\left.+\frac{k \sin k R}{R^{2}}\right)-\frac{k^{2} \cos ^{2} \phi \cos k R}{R}\right] \times \cos (l \Delta \psi),
\end{aligned}
$$

where $I$ and $\hbar c k$ denote the input irradiance and photon energy, respectively; $R$ is the interparticle displacement; and $\alpha$ is the dynamic polarizability. Also $f_{l p}$ and $A_{l p}$ represent the electric field radial function and normalization constant, respectively; and the angle between the polarization vector of the incoming beam and the interparticle displacement vector is defined as $\phi$. In the short-range region $(k R \ll 1)$, the leading term of Eq. (1), $\Delta E^{0}$, is found by taking the leading terms in the Taylor series expansions of $\sin k R$ and $\cos k R$. By use of the relation $R=\sqrt{2} r(1$ $-\cos \Delta \psi)^{1 / 2}, \Delta E^{0}$ is expressible as

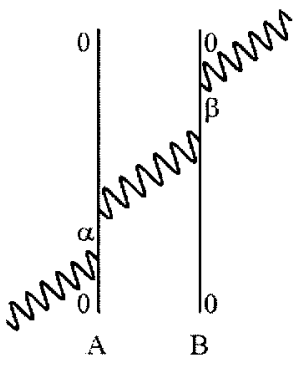

(a)

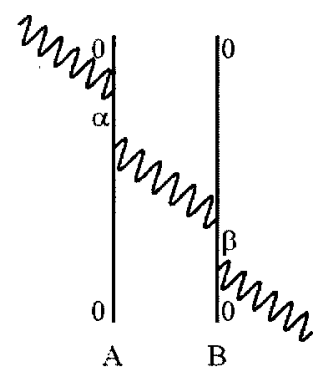

(b)
Fig. 1. Typical Feynman diagrams (each with 23 further time-ordered permutations) for calculation of the laserinduced interaction energy shift: 0 denotes the groundstate level, and $\alpha$ and $\beta$ are the excited levels for particles A and $\mathrm{B}$, respectively. 


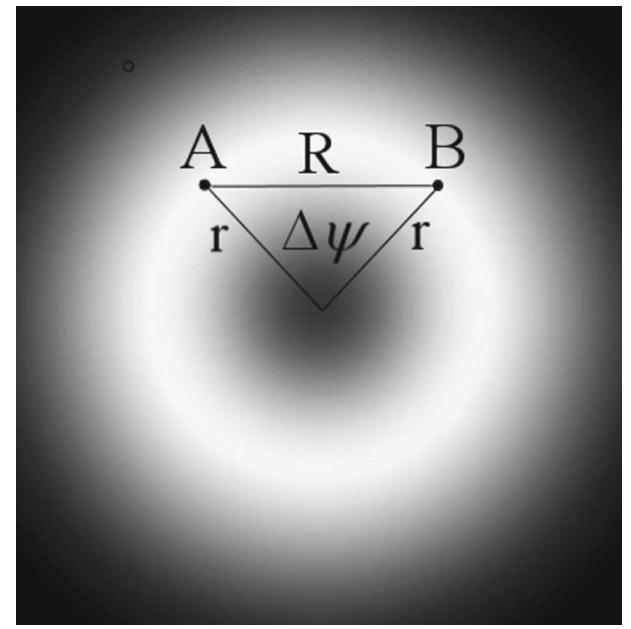

Fig. 2. Geometry of a spherical nanoparticle pair in a LG with $l=1, p=0$.
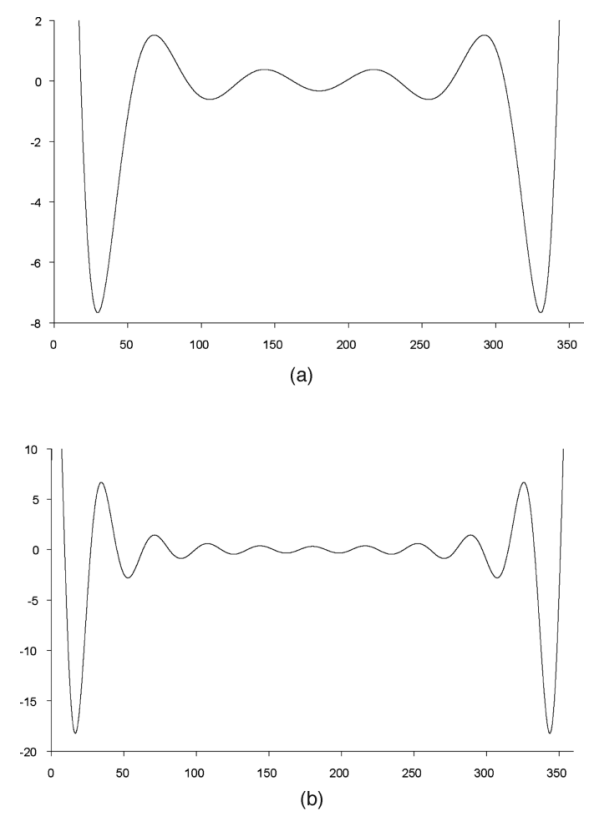

Fig. 3. Plot of $\Delta E_{\mathrm{AB}}^{0}$ versus $\Delta \psi$ for a nanoparticle pair in a LG beam with (a) $l=5$ and, (b) $l=10$.

$$
\Delta E_{\mathrm{AB}}^{0}=\left[\frac{I f_{l p}^{2} \alpha^{2}\left(1-3 \sin ^{2} \phi\right)}{8 \sqrt{2} \pi \epsilon_{0}^{2} r^{3} c A_{l p}}\right] \frac{\cos (l \Delta \psi)}{(\eta-\cos \Delta \psi)^{3 / 2}} .
$$

Here, $\eta$ is a damping factor whose introduction, in place of the unity that emerges from simple trigonometry, prevents $\Delta E_{\mathrm{AB}}^{0}$ rising nonphysically to infinity as $\Delta \psi$ approaches $0^{\circ}$ (the latter being a feature that would signify overlap of the particles). Treating the term inside the square brackets of Eq. (2) as constant, for the purpose of this work, graphs of $\Delta E_{\mathrm{AB}}^{0}$ against $\Delta \psi$ can be constructed for given values of $l$, as illustrated in Fig. 3. On detailed inspection of such graphs with varying $l$ it can be verified that (i) at $l$ $=0$, i.e., for a conventional Gaussian laser beam, the sole minimum is seen at $180^{\circ}$, illustrating that the energetically most favorable position of the particles in the beam cross section is where they are diametrically opposite each other (as expected); (ii) for odd values of $l$ greater than 1 , only a local minimum (not the energetically most favorable) arises for this configuration; (iii) for even values of $l$, a local maximum occurs at $180^{\circ}$; and (iv) generally, for $l \neq 0$, there are $l$ angular minima and $(l-1)$ maxima. Additional features reflect the behavior associated with increasing values of $l:(\mathrm{v})$ the number of positions for which the particle pair can be mutually trapped increases, becoming less energetically favorable as the angular disposition increases toward diametric opposition, and (vi) absolute minima are found at decreasing values of $\Delta \psi$ (Table 1), physically signifying a progression toward particle clustering. Note that the data in Table 1 (and those following) are determined with $\eta$ $=1$, i.e., no damping, as the results are sufficiently remote from values denoting particle overlap-the results are still within a few degrees of those produced by the graphs with $\eta=1.1$ damping accommodated.

Three- and four-particle systems. The two-particle analysis based on the configuration shown in Fig. 2 is easily extended to a three-particle arrangement. In this case $\Delta E_{\mathrm{ABC}}^{0}$ is determined by summing the pairwise laser-induced interactions of the three particles with each other, employing variables $\Delta \psi_{1}$ and $\Delta \psi_{2}$ as the azimuthal displacements between particles A-B and B-C, respectively. Here, $\Delta \psi_{1}$ and $\Delta \psi_{2}$ are in the interval $\left(0,180^{\circ}\right)$ and the corresponding angular disposition between $\mathrm{A}-\mathrm{C}$ is dependent on $\Delta \psi_{1}+\Delta \psi_{2}$ unless this exceeds $180^{\circ}$, in which case the dependence is $360^{\circ}-\left(\Delta \psi_{1}+\Delta \psi_{2}\right)$. Note that the term inside the square brackets in Eq. (2) gives the same values for the interaction of particles A-B, B-C, and A-C, except for its dependence on $\phi$; although this term varies for the differing directions of the interparticle displacement vector, the variation is slight and for simplicity is not accounted for. $3 \mathrm{D}$ plots of $\Delta E_{\mathrm{ABC}}^{0}$ versus $\Delta \psi_{1}$ and $\Delta \psi_{2}$ are exhibited in Fig. 4. Calculations of the absolute minima of $\Delta E_{\mathrm{ABC}}^{0}$ with $l=0$ attain the expected values of $120^{\circ}$ for both angles (equidistant particles). For higher values of $l$, as above, clustering is observed; for example, $l=4$ gives the values $\Delta \psi_{1}$ $=\Delta \psi_{2} \sim 30^{\circ}$.

Extending the analysis to a four-particle system, the corresponding energy shift $\Delta E_{\mathrm{ABCD}}^{0}$ is determined using a procedure similar to that outlined above with the additional variable $\Delta \psi_{3}$, the angle between particles $\mathrm{C}$ and $\mathrm{D}$. Determination of the minima of $\Delta E_{\mathrm{ABCD}}^{0}$ with $l=0$ confirms equidistant values of $90^{\circ}$ for each pair displacement angle; here the value $l$ $=4$ gives clustering with $\Delta \psi_{1} \sim 30^{\circ}, \Delta \psi_{2} \sim 29^{\circ}$, and $\Delta \psi_{3} \sim 30^{\circ}$. Physically, this demonstrates that the inner particle pair ( $\mathrm{B}$ and $\mathrm{C}$ ) clusters to a greater extent than the other pairs because of the additional influence of the outer particles (A and D). Further analysis of the three- and four-particle systems for varying values of $l$ reinforces the above features in

Table 1. Absolute Minima (in Degrees) of $\Delta E_{\mathrm{AB}}^{0}$ for Different $l$

\begin{tabular}{cccccc}
\hline$l$ & 0 & 2 & 4 & 10 & 20 \\
$\Delta \psi$ & 180 & 65.2 & 31.8 & 12.6 & 6.3 \\
\hline
\end{tabular}




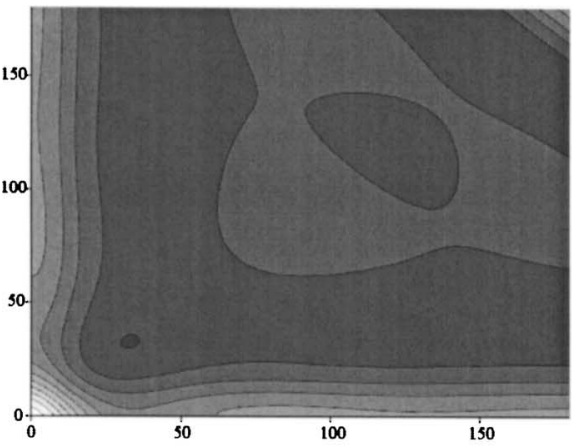

(a)

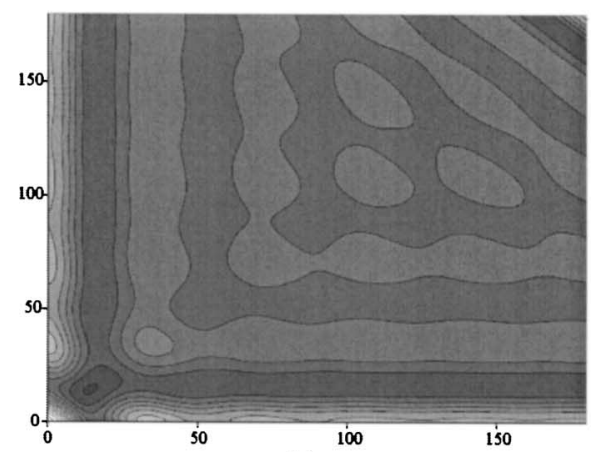

(b)

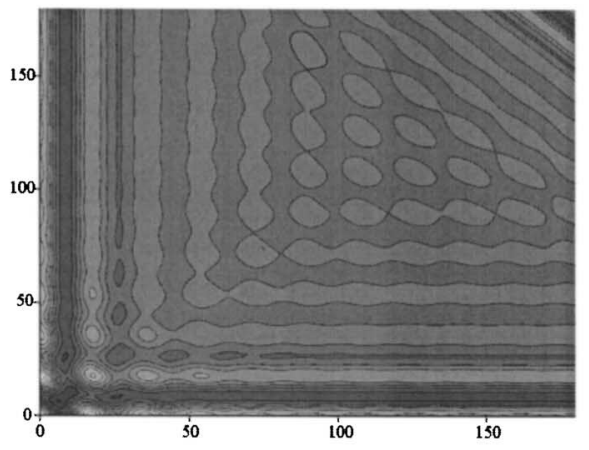

(c)

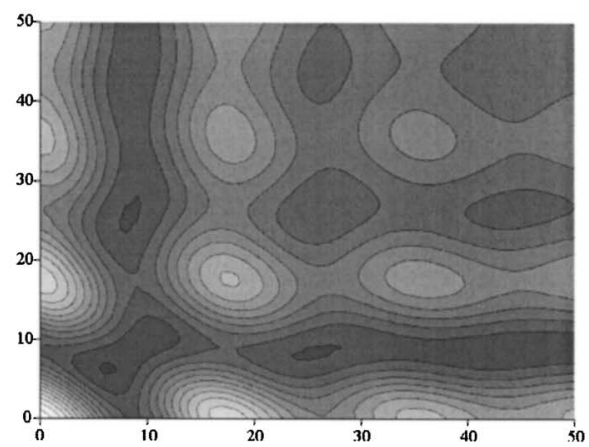

(d)

Fig. 4. Contour graph of $\Delta E_{\mathrm{ABC}}^{0}$ versus $\Delta \psi_{1}$ ( $x$ axis) and $\Delta \psi_{2}$ ( $y$ axis) for three nanoparticles in a LG beam with (a) $l=4$, (b) $l=10$, (c) $l=20$, (d) $l=20$ (close up). Lighter shading denotes higher values of $\Delta E_{\mathrm{ABC}}^{0}$.

that (i) $\Delta \psi_{1}, \Delta \psi_{2}$ for the three-particle case and $\Delta \psi_{1}$, $\Delta \psi_{3}$ for four particles give similar results, for example, $\sim 60^{\circ}$ for $l=2$ and $\sim 12^{\circ}$ for $l=10$; (ii) in comparison, $\Delta \psi_{2}$ is smaller for the physical reasons given above, for example, $\sim 56^{\circ}$ for $l=2$ and $\sim 11^{\circ}$ for $l=10$; and (iii) there is greater particle clustering for increasing $l$. It may be observed that three-body coupling between the particles is omitted from consideration in the above analysis. However, the complex analytical extension required for its inclusion ${ }^{10,11}$ is not justified since it involves higher-order perturbation theory and generates only insignificant corrections.

Although the results given above are exemplified by cases where $p=0$, the methods used also apply directly to cases with other values of $p$. Experimentally, however, there would be a technical problem in the possibility of particles being optically trapped in different annular intensity regions of the beam. LG modes with $p>0$ are in a sense similar in nature to higher-order Bessel modes, a type of beam also currently employed in optical tweezer systems. ${ }^{12} \mathrm{By}$ extension, theory can also be formulated for such beams-except that $f_{l p}$ in Eqs. (1) and (2) are substituted for an $l$ th-order Bessel function-where the same technical difficulty nonetheless arises if two or more annular regions are populated with nanoparticles.

The above findings illustrate that the laserinduced mechanisms operative here differ significantly from those of established optical spanner methods, not least because here there is no involvement of any orbital angular momentum transfer from the laser beam. It is hoped that future work by experimental groups will help identify the ordering and clustering features that we have identified.

The authors thank the UK Engineering and Physical Sciences Research Council for funding this work. D. L. Andrews' e-mail address is david.andrews @physics.org.

\section{References}

1. M. J. Padgett and L. Allen, Phys. World 10, 35 (1997).

2. M. M. Burns, J.-M. Fournier, and J. A. Golovchenko, Science 249, 749 (1990).

3. S. A. Tatarkova, A. E. Carruthers, and K. Dholakia, Phys. Rev. Lett. 89, 283901 (2002).

4. D. L. Andrews and D. S. Bradshaw, Opt. Lett. 30, 783 (2005).

5. L. C. Dávila Romero, D. L. Andrews, and M. Babiker, J. Opt. B: Quantum Semiclassical Opt. 4, S66 (2002).

6. N. B. Simpson, K. Dholakia, L. Allen, and M. J. Padgett, Opt. Lett. 22, 52 (1997).

7. A. Mair, A. Vaziri, G. Weihs, and A. Zeilinger, Nature (London) 412, 313 (2001).

8. D. S. Bradshaw and D. L. Andrews, in Proc. SPIE 5736, 87 (2005).

9. D. S. Bradshaw and D. L. Andrews, Phys. Rev. A 72, 033816 (2005).

10. D. P. Craig and T. Thirunamachandran, Chem. Phys. 135, 37 (1989).

11. R. Passante, E. A. Power, and T. Thirunamachandran, Phys. Rev. A 249, 77 (1998).

12. D. McGloin and K. Dholakia, Contemp. Phys. 46, 15 (2005). 